International Journal of Pure and Applied Mathematics

Volume 106 No. 1 2016, 59-74

ISSN: 1311-8080 (printed version); ISSN: 1314-3395 (on-line version)

url: http://www.ijpam.eu

doi: 10.12732/ijpam.v106i1.5

ijpam.eu

\title{
STOCHASTIC ANALYSIS OF PRINTED CIRCUIT BOARDS MANUFACTURING SYSTEM CONSIDERING REPAIRS BY INTERNAL/EXTERNAL ENGINEERS AND ARBITRARY ARRIVAL TIME OF EXTERNAL ENGINEERS
}

\author{
Shefali Batra ${ }^{1}$, Rajeev Kumar ${ }^{2}$ \\ ${ }^{1}$ Department of Mathematics \\ Chitkara University \\ Solan, Himachal Pradesh, INDIA \\ ${ }^{2}$ Department of Mathematics \\ Maharishi Dayanand University \\ Rohtak, Haryana, INDIA
}

\begin{abstract}
In the present paper, a stochastic model for the system involved in the printed circuit boards $(\mathrm{PCBs})$ manufacturing process is developed. The manufacturing process, based on Surface Mount Technology (SMT), is carried out in three stages viz. printing, placing and soldering through a hardware-software system. During the process, inspections, maintenances and repairs on occurrence of faults of the system are done by internal/external engineers. The internal engineers inspect the system and every manufactured PCB. The system's software faults as well as faults in the PCBs are repaired by the internal engineers whereas system's hardware faults are repaired only by the external engineers. In the model, possibilities of different kinds of faults, online PCB's repairs and arbitrary arrival time of the external engineers, are also incorporated. The expressions for various measures of system performance have been derived using Markov process and regenerative techniques. In the last, numerical computation and graphical analysis have been given for a particular case to show the effect of various parameters on the system performance and cost-benefit.
\end{abstract}

AMS Subject Classification: 60K15, 90B25, 60K10, 60J27

Key Words: printed circuit board (PCB), mean time to system failure (MTSF), availability, cost function, markov process and regenerative techniques

Received: August 7, 2015

Published: February 2, 2016

${ }^{\S}$ Correspondence author (c) 2016 Academic Publications, Ltd. url: www.acadpubl.eu 


\section{Introduction}

Many researchers have contributed extensively in the field of reliability. Several one and two units systems have been analysed by a large number of researchers under various conditions and situations. Nakagawa and Osaki (1975) considered a two-unit standby redundant system with repair and preventive maintenance. Yamashem (1980) worked on a multi-state system with several failure modes and cold standby unit. Goel et al. (1984) studied a two-unit cold standby system with two types of repair facilities. Goel and Murari (1990) discussed two-unit cold standby redundant system subject to random checking, corrective maintenance and system replacement with repairable and non-repairable types of failure. Gopalan and Bhanu (1995) studied two unit repairable system subject to on-line preventive maintenance and/or repair. Gupta et al. (1997) analysed a system having super-priority, priority and ordinary units with arbitrary distributions. Wang and Kuo (2000) studied cost and probabilistic analysis of series system with mixed standby components. Kumar et al. (2001) discussed a two-unit redundant system with degradation replacement. Taneja and Nanda (2003) analysed a two-unit cold standby system with resume and repeat repair policies. Taneja et al. (2006) discussed economic analysis of a reliability model for two-programmable logic controller cold standby system with four types of failures. Xing (2007) proposed efficient methods to assess the reliability of phased-mission systems considering both imperfect fault coverage and common-cause failures. Goyal et al. (2009) analysed a two unit cold standby system working in a sugar mill with operating and rest period. Mahmoud and Moshref (2010) studied a two-unit cold standby system considering hardware, human error failures and preventive maintenance. Kumar and Bhatia (2011) discussed reliability and cost analysis of one-unit centrifuge system with single repairman and inspection. Kumar and Malik (2012) studied reliability modelling of a computer system with priority to software replacement over hardware replacement subject to maximum operation time and maximum repair time. Kumar and Kumar (2012) studied performance and cost benefit analysis of a hardware-software system considering hardware based software interaction failures and different types of recovery. Mohammed (2012) discussed availability of a system with different repair options. Jain and Rani (2013) analysed availability for repairable system with warm standby, switching failure and reboot delay. Wells (2014) analysed reliability of a single warm-standby system subject to repairable and non-repairable failures. The PCBs manufacturing process based on surface mount technology plays very important role in the electronics industry as PCBs are very widely used as the basis for all elec- 
tronic circuits/equipments. There are a number of individual stages involved in PCBs manufacturing process system, however it is necessary for all of them to work together to form an overall integrated process. For failure-free and cost effective performance of PCBs manufacturing process and further in-depth study of reliability and cost of a $\mathrm{PCB}$, a PCBs manufacturing plant located at Panchkula (Haryana) was visited and the relevant information/ data regarding working process, types of faults, repairs, maintenances, inspection etc. of the system was collected.The various stages in the PCBs manufacturing process comprises adding solder paste to the board, pick and place of the components, soldering and inspections. All these stages are required to be monitored to ensure highest quality of the manufactured product. First stage in the manufacturing process is solder paste, in which solder paste is added to those areas of the board where solder is required prior to the addition of the components to the board. After this step, the board is passed through pick and place stage in which required components are placed at correct position. In the next stage, the board is passed through a soldering machine. Under soldering machine, the PCB undergoes many temperature variations to ensure the connectivity of joints. All these stages involve hardware as well as software operations. Additionally, the operation of the system needs some start up time and then set up time to manufacture a particular type of PCB.The system may fail due to various hardware/software faults namely Pick and place, Stencil Printer,Reflow Oven, Software and Utility Services like improper illumination, failure of electricity etc. From the collected data,following estimates are obtained.

Pick and Place failure rate $\left(\lambda_{1}\right)$

Printer failure $\operatorname{rate}\left(\lambda_{2}\right)$

Reflow oven failure rate $\left(\lambda_{3}\right)$

Software failure rate $\left(\lambda_{4}\right)$

Utility Services failure rate $\left(\lambda_{5}\right)$

Shutdown $\operatorname{rate}(\eta)$

Preventive maintenance rate $(\alpha)$

Rate at which PCB goes for inspection $(\gamma)$

$\mathrm{PCB}$ inspection $\operatorname{rate}\left(\psi_{2}\right)$

$$
\begin{aligned}
& =0.0041 / \mathrm{hr} \\
& =0.0006 / \mathrm{hr} \\
& =0.0040 / \mathrm{hr} \\
& =0.0005 / \mathrm{hr} \\
& =0.0010 / \mathrm{hr} \\
& =0.00747 / \mathrm{hr} \\
& =0.156956 / \mathrm{hr} \\
& =17 / \mathrm{hr} \\
& =17 / \mathrm{hr}
\end{aligned}
$$

On the basis of the collected information/data for the PCBs manufacturing process, Kumar and Batra (2012) developed model considering two types of repair facilities-internal engineers and external engineers. It was assumed that on failure of a unit, internal engineers first inspect the type of fault and then 
inspect whether the fault is repairable at internal level or not. If the system is repairable, the internal engineer carries out repairs/replacements; otherwise the external engineer is called for the same. Moreover if infected PCB's are found that are repaired offline. This is not a general model that takes care of all practical aspects/situations for the system such as possibilities of different rates/kinds of faults, online PCB's repairs, arbitrary arrival time of the external engineers etc.

Thus the aim of the present paper is to develop a more general and practically viable model for the system, implementation of which in turns leads to better performance with lesser cost to the stakeholders. Keeping this in view, in this paper, a stochastic model for the system incorporating realistic and practical situations is developed. In the model, rate of occurrence of different kinds of faults in the system are considered. The internal engineers inspect the system and each manufactured PCB. On the failure of system, he inspects the system to assess whether there are hardware faults or software faults. It is considered that the hardware faults are repaired by the external engineers whereas other faults like utility services faults, software faults, faults in the PCBs are repaired by the internal engineers. As it was observed that in Pick and Place, Stencil Printer, and Reflow Oven components, hardware faults are repaired by the external engineers whereas software faults in these, besides other faults, are repaired by the internal engineers. Further aspects of arbitrary arrival time of the external engineers and online PCB's repair by internal engineers have also been incorporated. Expressions for various measures of system performance such as mean time to system failure (MTSF), availability, busy period of the internal/external engineers for PCB/process inspections and repairs/replacements, expected numbers of repairs and visits of the external engineers are derived for the model by making use of Markov process and regenerative point techniques. The system is analysed in terms of mean time to system failure, availability and cost-benefit by plotting graphs for a particular case.

\subsection{Other Assumptions}

1. The system is initially operative.

2. Faults are self-announcing.

3. Each PCB is inspected to assess whether it is as per required specification or not.

4. The system is as good as new after the repair.

5. The system is periodically shut down for preventive maintenance.

6. The times to failure, shutdown, preventive maintenance, PCB goes for 
inspection follow exponential distribution whereas other times follow general distributions.

\subsection{States of System}

O Operative State;

$O_{i_{2}} \quad$ Operative state and PCB under inspection;

$\mathrm{O}_{S w_{r}} \quad$ Operative state and PCB software fault under online repair;

$\mathrm{R} \quad$ Halt state for preventive maintenance;

$F_{P_{P i_{3}}} \quad$ Failed state due to Pick and Place fault and under inspection;

$F_{\mathrm{PTi}_{4}} \quad$ Failed state due to Printer fault and under inspection;

$F_{R O i_{5}} \quad$ Failed state due to Reflow Oven fault and under inspection;

$F_{S w_{r}} \quad$ Failed state due to Software fault and under repair;

$F_{U S_{r}} \quad$ Failed state due to Utility Services fault and under repair;

$F_{P P H W} \quad$ Failed state due to Pick and Place hardware fault and; under wait;

$F_{P P H W_{r}} \quad$ Failed state due to Pick and Place hardware fault and repair;

$F_{P P S W_{r}} \quad$ Failed state due to Pick and Place software fault and under repair;

$F_{P T H W} \quad$ Failed state due to Printer hardware fault and under wait;

$F_{P T H W_{r}} \quad$ Failed state due to Printer hardware fault and under repair;

$F_{P P S W_{r}} \quad$ Failed state due to Printer software fault and under repair;

$F_{R O H W} \quad$ Failed state due to Reflow hardware fault and under wait;

$F_{R O H W_{r}} \quad$ Failed state due to Reflow hardware fault and under repair;

$F_{P P S W_{r}} \quad$ Failed state due to Reflow software fault and under repair.

\subsection{Nomenclature}

$\lambda_{1} / \lambda_{2} / \lambda_{3} \quad$ Pick and Place/Printer/Reflow Oven failure rate;

$\lambda_{4} / \lambda_{5}$

$\eta$

$\alpha$

$\gamma$

$p_{1}$

$p_{2}$

$p_{4} / q_{4}$

Software Utility Services failure rate;

Shutdown rate for preventive maintenance;

Preventive maintenance rate;

Rate at which PCB goes for inspection;

Probability that PCB is found O.K.;

Probability that PCB has software fault;

$p_{5} / q_{5}$

Probability of Pick and Place hardware/ software fault;

$p_{6} / q_{6}$

Probability of Printer hardware/ software fault;

$g_{1}(t) / G_{1}(t)$

Probability of Reflow Oven hardware/software fault;

p.d.f./c.d.f of repair time for Pick and Place hardware fault; 
$g_{2}(t) / G_{2}(t) \quad$ p.d.f./c.d.f of repair time for Printer hardware fault;

$g_{3}(t) / G_{3}(t) \quad$ p.d.f./c.d.f of repair time for Reflow Oven hardware fault;

$g_{4}(t) / G_{4}(t) \quad$ p.d.f./c.d.f of repair time for Software fault;

$g_{5}(t) / G_{5}(t) \quad$ p.d.f./c.d.f of repair time for Utility Services fault;

$g_{6}(t) / G_{6}(t) \quad$ p.d.f./c.d.f of repair time for Pick and Place software fault;

$g_{7}(t) / G_{7}(t) \quad$ p.d.f/c.d.f of repair time for Printer software fault;

$g_{8}(t) / G_{8}(t) \quad$ p.d.f/c.d.f of repair time for Reflow Oven software fault;

$g_{9}(t) / G_{9}(t) \quad$ p.d.f/c.d.f of repair time for PCB faults;

$i_{2}(t) / I_{2}(t) \quad$ p.d.f./c.d.f of PCB inspection time;

$i_{3}(t) / I_{3}(t) \quad$ p.d.f./c.d.f of Pick and Place fault inspection time;

$i_{4}(t) / I_{4}(t) \quad$ p.d.f/c.d.f of Printer fault inspection time;

$i_{5}(t) / I_{5}(t) \quad$ p.d.f/c.d.f of Reflow Oven fault inspection time;

$\mathrm{k}(\mathrm{t}) / \mathrm{K}(\mathrm{t}) \quad$ p.d.f/c.d.f of arbitrary arrival time of external engineer.

\subsection{State Transition Diagram}

The state transition diagram in Figure 1 which shows the epoch of entries into the states $0,1,2,3,4,5,6,7,8,9,10,11,12,13,14,15,16$ and 17 are regenerative points and thus these are considered as regenerative states. The states other than ' 0 ' are failed states.

\section{Transition Probabilities and Mean Sojourn Times}

Let $q_{i j}(t)$ represents probability density function (p.d.f.) of first passage time from a regenerative state $\mathrm{i}$ to $\mathrm{j}$ or to failed state $\mathrm{j}$ without visiting any other regenerative state in $(0, \mathrm{t}]$. Then transition probabilities are given by

$$
\begin{array}{ll}
q_{01}(t)=\lambda_{1} e^{-\left(\lambda_{1}+\lambda_{2}+\lambda_{3}+\lambda_{4}+\lambda_{5}+\eta+\gamma\right) t}, & q_{02}(t)=\lambda_{2} e^{-\left(\lambda_{1}+\lambda_{2}+\lambda_{3}+\lambda_{4}+\lambda_{5}+\eta+\gamma\right) t}, \\
q_{03}(t)=\lambda_{3} e^{-\left(\lambda_{1}+\lambda_{2}+\lambda_{3}+\lambda_{4}+\lambda_{5}+\eta+\gamma\right) t}, & q_{04}(t)=\lambda_{4} e^{-\left(\lambda_{1}+\lambda_{2}+\lambda_{3}+\lambda_{4}+\lambda_{5}+\eta+\gamma\right) t}, \\
q_{05}(t)=\lambda_{5} e^{-\left(\lambda_{1}+\lambda_{2}+\lambda_{3}+\lambda_{4}+\lambda_{5}+\eta+\gamma\right) t}, & q_{06}(t)=\eta e^{-\left(\lambda_{1}+\lambda_{2}+\lambda_{3}+\lambda_{4}+\lambda_{5}+\eta+\gamma\right) t}, \\
q_{07}(t)=\gamma e^{-\left(\lambda_{1}+\lambda_{2}+\lambda_{3}+\lambda_{4}+\lambda_{5}+\eta+\gamma\right) t}, &
\end{array}
$$

$q_{1,9}(t)=p_{4} i_{3}(t), q_{1,11}(t)=q_{4} i_{3}(t), q_{9,10}(t)=k(t), q_{2,12}(t)=p_{5} i_{4}(t), q_{2,14}(t)=$ $q_{5} i_{4}(t), q_{12,13}(t)=k(t), q_{3,15}(t)=p_{6} i_{5}(t), q_{3,17}(t)=q_{6} i_{5}(t), q_{15,16}(t)=k(t)$, $q_{4,0}(t)=g_{4}(t), q_{5,0}(t)=g_{5}(t), q_{6,0}(t)=\alpha e^{-\alpha t}, q_{7,0}(t)=p_{1} i_{2}(t), q_{7,8}(t)=$ $p_{2} i_{2}(t), q_{8,0}(t)=g_{9}(t), q_{10,0}(t)=g_{1}(t), q_{11,0}(t)=g_{6}(t), q_{13,0}(t)=g_{2}(t)$, $q_{14,0}(t)=g_{7}(t), q_{16,0}(t)=g_{3}(t), q_{17,0}(t)=g_{8} i(t)$. 

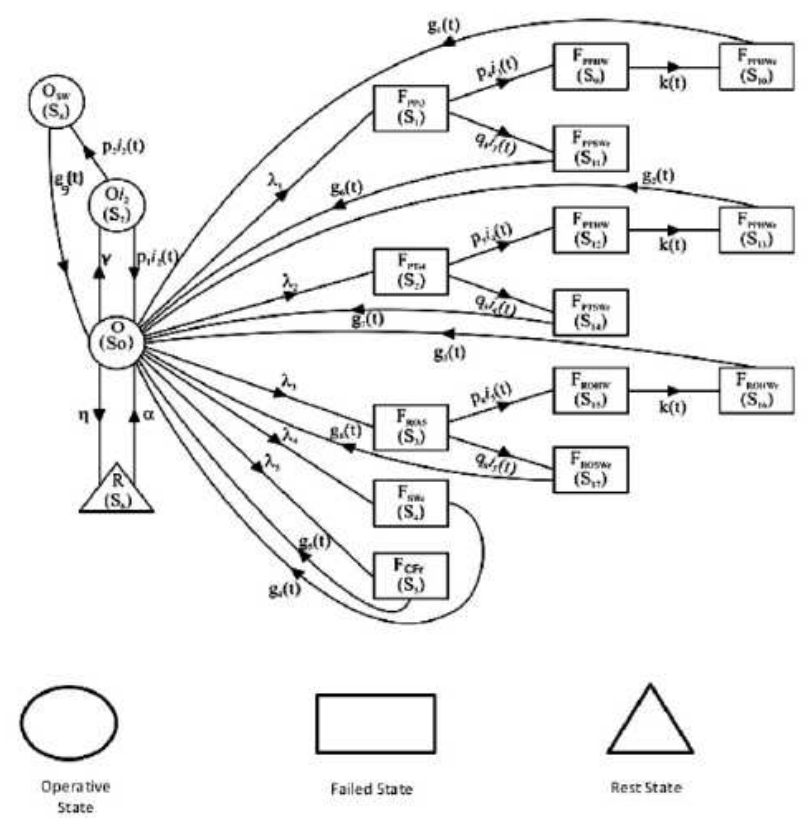

Figure 1

The non-zero elements $p_{i j}=\lim _{t \rightarrow \infty} q_{i j}(t)$ are

$$
\begin{gathered}
p_{01}=\frac{\lambda_{1}}{\lambda_{1}+\lambda_{2}+\lambda_{3}+\lambda_{4}+\lambda_{5}+\eta+\gamma}, p_{02}=\frac{\lambda_{2}}{\lambda_{1}+\lambda_{2}+\lambda_{3}+\lambda_{4}+\lambda_{5}+\eta+\gamma}, \\
p_{03}=\frac{\lambda_{3}}{\lambda_{1}+\lambda_{2}+\lambda_{3}+\lambda_{4}+\lambda_{5}+\eta+\gamma}, p_{04}=\frac{\lambda_{4}}{\lambda_{1}+\lambda_{2}+\lambda_{3}+\lambda_{4}+\lambda_{5}+\eta+\gamma}, \\
p_{05}=\frac{\eta}{\lambda_{1}+\lambda_{2}+\lambda_{3}+\lambda_{4}+\lambda_{5}+\eta+\gamma}, p_{06}=\frac{\eta}{\lambda_{1}+\lambda_{2}+\lambda_{3}+\lambda_{4}+\lambda_{5}+\eta+\gamma}, \\
p_{07}=\frac{\lambda_{3}}{\lambda_{1}+\lambda_{2}+\lambda_{3}+\lambda_{4}+\lambda_{5}+\eta+\gamma}, \\
p_{1,9}=p_{4}, p_{1,11}=q_{4}, p_{2,12}=p_{5}, p_{2,14}=q_{5}, \\
p_{3,15}=p_{6}, p_{3,17}=q_{6}, \quad p_{7,8}=p_{2}, \quad p_{7,0}=p_{1}, \\
p_{9,10}=p_{8,0}=p_{10,0}=p_{11,0}=p_{12,13}=p_{4,0}=p_{5,0}=p_{6,0}=p_{13,0}=1, \\
p_{14,0}=p_{15,16}=p_{16,0}=p_{17,0}=1 .
\end{gathered}
$$


The mean sojourn times $\left(\mu_{i}\right)$ are obtained as

$$
\begin{array}{llll}
\mu_{0}=\frac{1}{\lambda_{1}+\lambda_{2}+\lambda_{3}+\lambda_{4}+\lambda_{5}+\eta+\gamma}, & \mu_{1}=\int_{0}^{\infty} \overline{I_{3}(t)} d t, & \mu_{2}=\int_{0}^{\infty} \overline{I_{4}(t)} d t \\
m u_{3}=\int_{0}^{\infty} \overline{I_{5}(t)} d t, & \mu_{4}=\int_{0}^{\infty} \overline{G_{4}(t)} d t, & \mu_{5}=\int_{0}^{\infty} \overline{G_{5}(t)} d t, \\
\mu_{6}=\frac{1}{\alpha}, & \mu_{7}=\int_{0}^{\infty} \overline{I_{2}(t)} d t, & \mu_{8}=\int_{0}^{\infty} \overline{G_{9}(t)} d t, \\
\mu_{9}=\mu_{12}=\mu_{15}=\int_{0}^{\infty} \overline{K(t)} d t, & \mu_{10}=\int_{0}^{\infty} \overline{G_{1}(t)} d t, & \\
\mu_{11}=\int_{0}^{\infty} \overline{G_{6}(t)} d t, & \mu_{13}=\int_{0}^{\infty} \overline{G_{2}(t)} d t, & \mu_{14}=\int_{0}^{\infty} \overline{G_{7}(t)} d t, \\
\mu_{16}=\int_{0}^{\infty} \overline{G_{3}(t)} d t, & \mu_{17}=\int_{0}^{\infty} \overline{G_{8}(t)} d t .
\end{array}
$$

The unconditional mean time taken by the system to transit for any state ' $\mathrm{j}$ ' when it is counted from epoch of entrance into state ' $\mathrm{i}$ ' is, mathematically, stated as

$$
m_{i j}=\int_{0}^{\infty} t q_{i j}(t) d t=-q_{i j}^{*^{\prime}}(0) .
$$

Thus

$$
\begin{aligned}
& m_{01}+m_{02}+m_{03}+m_{04}+m_{05}+m_{06}+m_{07}=\mu_{0}, \quad m_{1,9}+m_{1,11}=\mu_{1}, \\
& m_{2,12}+m_{2,14}=\mu_{2}, \quad m_{3,15}+m_{3,17}=\mu_{3}, \quad m_{40}=\mu_{4}, \quad m_{50}=\mu_{5}, \\
& m_{60}=\mu_{6}, \quad m_{70}+m_{78}=\mu_{7}, \quad m_{8,0}=\mu_{8}, \quad m_{9,10}=\mu_{9} \text {, } \\
& m_{10,0}=\mu_{10}, \quad m_{11,0}=\mu_{11}, \quad m_{12,13}=\mu_{12}, m_{13,0}=\mu_{13} \text {, } \\
& m_{14,0}=\mu_{14}, \quad m_{15,16}=\mu_{15}, \quad m_{16,0}=\mu_{16}, \quad m_{17,0}=\mu_{17} \text {, }
\end{aligned}
$$

\section{Other Measures of System Performance}

Using probabilistic arguments for regenerative process, various recursive relations are expressed and solved to drive measures of system effectiveness. Various measures of system effectiveness obtained are as under:

Mean time to system

failure $\left(M_{0}\right)$

Steady state availability

$$
\begin{aligned}
& =\frac{\mu_{0}+p_{06} \mu_{6}+p_{07} \mu_{7}+p_{07} p_{2} \mu_{8}}{1-p_{06}-p_{07}} \\
& =\frac{\mu_{0}+p_{07} \mu_{7}+p_{07} p_{2} \mu_{8}}{D}
\end{aligned}
$$

Busy period of the internal engineers 
(Fault inspection only) $\left(B I_{0}\right) \quad=\frac{\mu_{1} p_{01}+p_{02} \mu_{2}+p_{03} \mu_{3}}{D}$

Busy period of the internal engineers

(Repair time only) $\left(B R_{0}\right)=\frac{\mu_{11} p_{01} p_{1,11}+p_{02} \mu_{14} p_{2,14}+p_{03} p_{3,17} \mu_{17}+p_{07} p_{7,8} \mu_{8}+p_{05} \mu_{5}+p_{04} \mu_{4}}{D}$ Busy period of the internal engineers

$\left(\mathrm{PCB}\right.$ inspection time only) $\left(B P_{0}\right)=\frac{p_{07} \mu_{7}}{D}$

Expected number of repairs

by the internal engineers $\left(R I_{0}\right) \quad=\frac{p_{01} p_{1,11}+p_{02} p_{2,14}+p_{03} p_{3,17}+p_{07} p_{7,8}+p_{05}+p_{04}}{D}$

Busy period of the external engineers

(Repair time only) $\left(B E_{0}\right) \quad=\frac{p_{01} \mu_{1} p_{1,9}+p_{02} \mu_{2} p_{2,12}+p_{03} \mu_{3} p_{3,15}}{D}$

Expected number of repairs

by the external engineers $\left(R E_{0}\right)=\frac{p_{01} p_{1,9}+p_{02} p_{2,12}+p_{03} p_{3,15}}{D}$

Expected number of visits of the

external engineers $\left(V E_{0}\right) \quad=\frac{p_{01} p_{1,9}+p_{02} p_{2,12}+p_{03} p_{3,15}}{D}$,

where

$$
\begin{gathered}
D=\mu_{0}+p_{01}\left(\mu_{1}+p_{1,9}\left(\mu_{9}+\mu_{10}\right)+p_{1,11} \mu_{11}\right)+p_{02}\left(\mu_{2}+p_{2,12}\left(\mu_{12}+\mu_{13}\right)+p_{2,14} \mu_{14}\right) \\
+p_{03}\left(\mu_{3}+p_{3,15}\left(\mu_{15}+\mu_{16}\right)+p_{3,17} \mu_{17}\right)+\mu_{4} p_{04}+\mu_{5} p_{05}+\mu_{6} p_{06}+p_{07}\left(\mu_{7}+\mu_{8} p_{78}\right) .
\end{gathered}
$$

\section{Profit Analysis}

The profit incurred to the system in steady state can be obtained as

$P=C_{0} A_{0}-C_{1} B I_{0}-C_{2} B R_{0}-C_{3} R I_{0}-C_{4} R E_{0}-C_{5} B E_{0}-C_{6} V E_{0}-C_{7}-C_{8} B P_{0}$,

where

$C_{0}=$ Revenue per unit uptime of system;

$C_{1}=$ Cost per unit time of fault inspection;

$C_{2}=$ Cost per unit time of internal repair;

$C_{3}=$ Cost per unit repair by internal engineer;

$C_{4}=$ Cost per unit repair by external engineer;

$C_{5}=$ Cost per unit time of external repair;

$C_{6}=$ Cost per visit of external engineer;

$C_{7}=$ Other fixed costs;

$C_{8}=$ Cost per unit time of PCB inspection. 


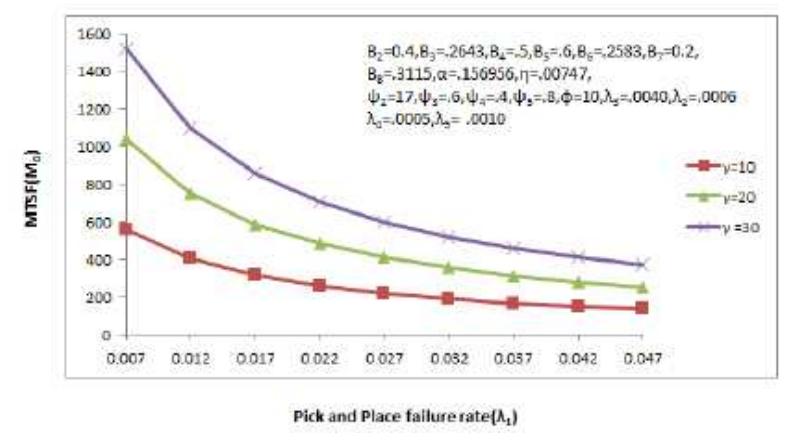

Figure 2: Mean Time to System Failure $\left(M_{0}\right)$ Versus Pick and Place Failure Rate $\left(\lambda_{1}\right)$ For Different Values of Rate at which PCB goes for Inspection $(\gamma)$

\section{Numerical Computation and Graphical Analysis}

Numerical computation for the following particular case is carried out and various graphs are drawn:

$$
\begin{array}{lll}
g_{1}(t)=\beta_{1} e^{-\beta_{1}(t)}, & g_{2}(t)=\beta_{2} e^{-\beta_{2}(t)}, & g_{3}(t)=\beta_{3} e^{-\beta_{3}(t)}, \\
g_{4}(t)=\beta_{4} e^{-\beta_{4}(t)}, & g_{5}(t)=\beta_{5} e^{-\beta_{5}(t)}, & g_{6}(t)=\beta_{6} e^{-\beta_{6}(t)} \\
g_{7}(t)=\beta_{7} e^{-\beta_{7}(t)}, & g_{8}(t)=\beta_{8} e^{-\beta_{8}(t)}, & g_{9}(t)=\beta_{9} e^{-\beta_{9}(t)}, \\
k(t)=\phi e^{-\phi(t)}, & i_{2}(t)=\psi_{2} e^{-\psi_{2}(t)}, & i_{3}(t)=\psi_{3} e^{-\psi_{3}(t)}, \\
i_{4}(t)=\psi_{4} e^{-\psi_{4}(t)}, & i_{5}(t)=\psi_{5} e^{-\psi_{5}(t)} &
\end{array}
$$

Various graphs are plotted for the mean time to system failure $\left(M_{0}\right)$, availability $\left(A_{0}\right)$ and profit $(P)$ of the system for the model for different values of failure rates $\left(\lambda_{1}, \lambda_{2}, \lambda_{3}, \lambda_{4}, \lambda_{5}\right)$, repair rates $\left(\beta_{1}, \beta_{2}, \beta_{3}, \beta_{4}, \beta_{5}, \beta_{6}, \beta_{7}, \beta_{8}, \beta_{9}\right)$, inspection rates $\left(\psi_{2}, \psi_{3}, \psi_{4}, \psi_{5}\right)$ and arbitrary arrival rate $(\phi)$. The following conclusion has been drawn from the graphs:

Figure 2 depicts the behaviour of mean time to system failure $\left(M_{0}\right)$ with respect to the pick and place failure rate $\left(\lambda_{1}\right)$ for different values of rate at which PCB goes for inspection $(\gamma)$. It can be observed from the graph that as Pick and Place failure rate increases the mean time to system failure decreases and further, mean time to system failure has higher values for higher values of the rate at which PCB goes for inspection $(\gamma)$.

The graph in Figure 3 depicts the behaviour of availability $\left(A_{0}\right)$ with respect to Pick and Place failure rate $\left(\lambda_{1}\right)$ for different values of Pick and Place repair 


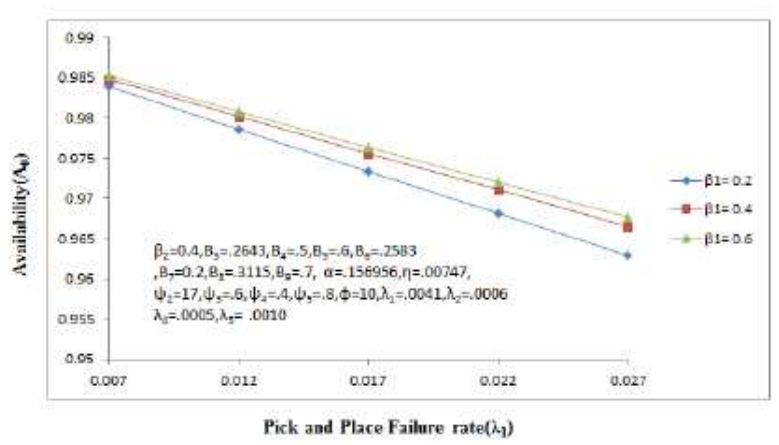

Figure 3: Availability $\left(A_{0}\right)$ Versus Pick and Place Failure Rate $\left(\lambda_{1}\right)$ For Different Values of Pick and Place Repair Rate $\left(\beta_{1}\right)$

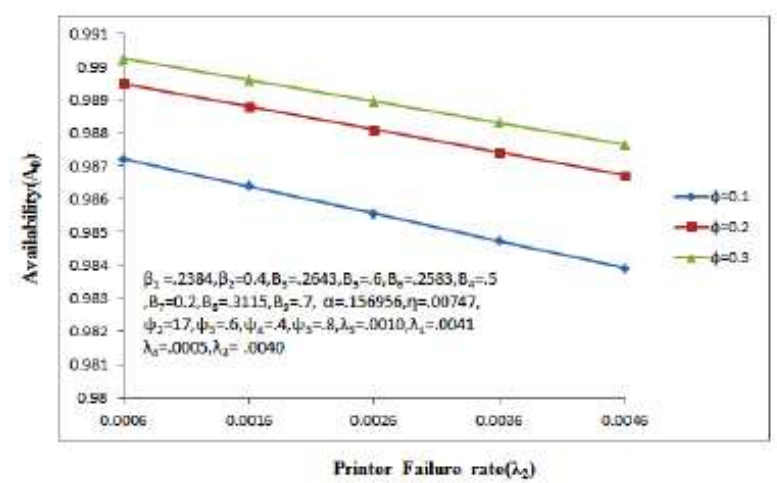

Figure 4: Availability $\left(A_{0}\right)$ Versus Printer Failure Rate $\left(\lambda_{2}\right)$ For Different Values of Arrival Rate of External Engineer $(\phi)$

rate $\left(\beta_{1}\right)$. It can be observed from the graph that availability of the system decreases with increase in the values of Pick and place failure rate and has higher values for higher values of Pick and Place repair rate. The graph in Figure 4 depicts the behaviour of availability $\left(A_{0}\right)$ with respect to Printer failure rate $\left(\lambda_{2}\right)$ for different values of arbitrary arrival rate of external engineer $(\phi)$. It can be observed from the graph that availability of the system decreases with increase in the values of Printer failure rate and also increases with increase in values of arrival rate of external engineer.

The graph in Figure 5 depicts the behaviour of availability $\left(A_{0}\right)$ with respect to Reflow Oven failure rate $\left(\lambda_{3}\right)$ for different values of Reflow Oven fault inspection rate $\left(\psi_{5}\right)$. It can be observed from the graph that availability of the 


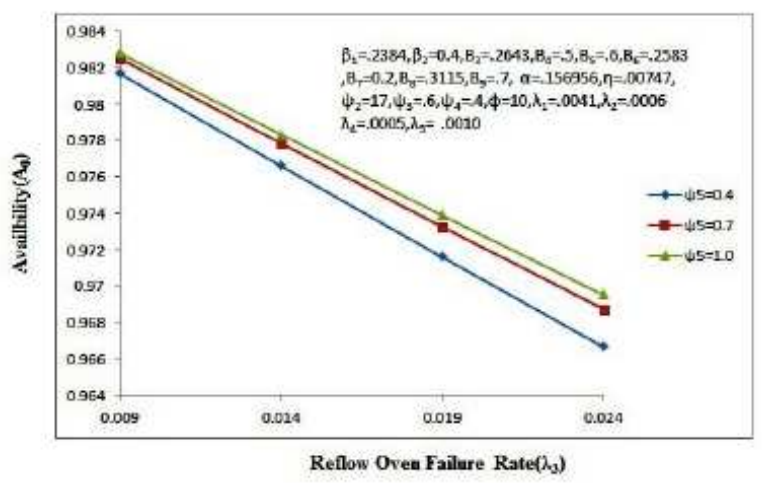

Figure 5: Availability $\left(A_{0}\right)$ Versus Reflow Oven Failure Rate $\left(\lambda_{3}\right)$ For Different Values of Reflow Oven Fault Inspection Rate $\left(\psi_{5}\right)$

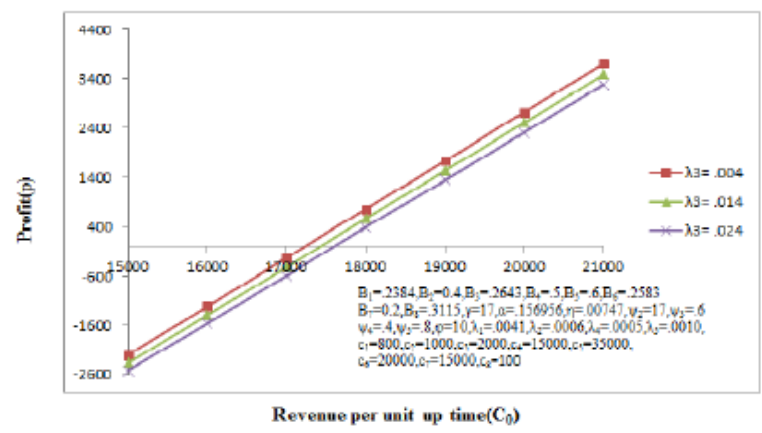

Figure 6: Profit $(P)$ Versus Revenue per Unit Uptime $\left(C_{0}\right)$ For Different Values of Reflow Oven Failure Rate $\left(\lambda_{3}\right)$

system decreases with increase in the values of Reflow Oven failure rate and has higher values for higher values of Reflow Oven fault inspection rate.

The curves in Figure 6 show the pattern of profit $(P)$ with respect to revenue per unit uptime $\left(C_{0}\right)$ for different values of Reflow Oven failure rate $\left(\lambda_{3}\right)$ of system. It can be concluded from the graph that profit increases with increase in revenue per unit uptime of system and further it decreases with the increase in values of Reflow Oven failure rate. For Reflow Oven failure rate $\left(\lambda_{3}\right)=0.004$, $\mathrm{P}<0$ or $=0$ or $>0$, according as $\left(C_{0}\right)<$ or $=$ or $>17236.66$. Therefore for the profit, the revenue per unit uptime of the system $\left(C_{0}\right)$ should be $>17236.66$. Similarly, for Reflow Oven failure rate $=0.014$ and 0.024 , for the profits, $\left(C_{0}\right)$ $>17423.62$ and 17610.5 respectively. The curves in Figure 7 show the pattern 


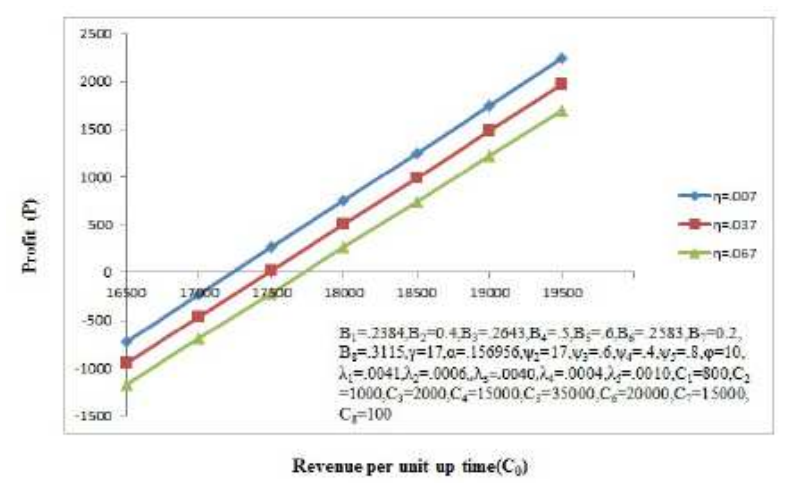

Figure 7: Profit $(\mathrm{P})$ Versus Revenue per unit Uptime $\left(C_{0}\right)$ For Different Values of Shutdown Rate $(\eta)$

of profit $(P)$ with respect to revenue per unit uptime $\left(C_{0}\right)$ for different values of shut down rate $(\eta)$ of system. It can be concluded from the graph that as revenue per unit uptime of system increases, the profit increases and further it decreases with the increase in values of shut down rate. For shutdown rate $(\eta)=0.007, \mathrm{P}<0$ or $=0$ or $>0$, according as $\left(C_{0}\right)<$ or $=$ or $>17232.8$. Therefore for the profit, the revenue per unit uptime of the system $\left(C_{0}\right)$ should be $>17232.8$. Similarly, for shutdown rate $=0.037$ and 0.067 , for the profits, $\left(C_{0}\right)>17477.5$ and 17722.31 respectively.

Figure 8 shows the behaviour of profit $(P)$ with respect to cost per unit time of external repair $\left(C_{5}\right)$ for different values of Printer failure rate $\left(\lambda_{2}\right)$. It can be concluded that as cost per unit time of external repair increases profit incurred for the system decreases and further it decreases with increase in values of Printer failure rate. For Printer failure rate $\left(\lambda_{2}\right)=0.0005, \mathrm{P}<0$ or $=0$ or $>$ 0 , as $C_{5}>$ or $=$ or $<128879.5$. Therefore for the profit, the cost per unit time of external repair of the system $C_{5}$ should be less than 128879.5. Similarly, for Printer failure rates $\left(\lambda_{2}\right)=0.0015$ and 0.0025 , for the profits, $C_{5}$ should be less than 98081 and 72526.5 respectively.

\section{Conclusion}

The optimal trade-off between quality and cost must be agreed upon by the manufacturer and the customer to ensure overall expectation of the deliverables is achieved. On the basis of realistic data and estimated parameters a stochastic model for PCB manufacturing process is proposed by considering two types of 


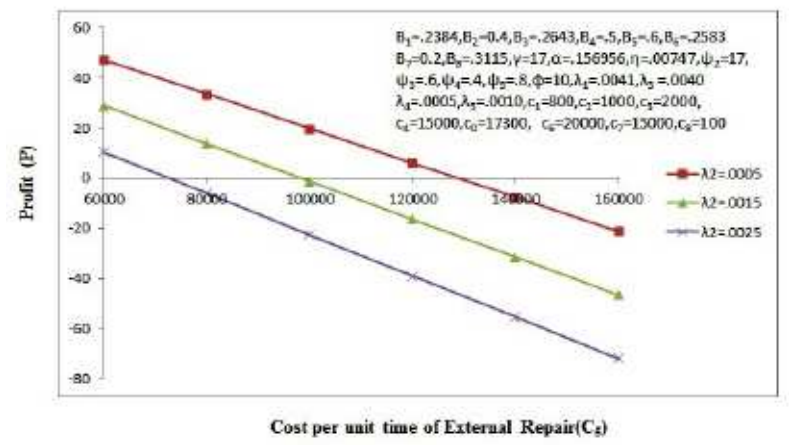

Figure 8: Profit (P) Versus Cost per unit time of External Repair $\left(C_{5}\right)$ For Different Values of Printer Failure Rate $\left(\lambda_{2}\right)$

repair facilities and delay in arrival of external engineers. Various measures of performance and profit incurred for the system are obtained. These measures have been analysed graphically in terms of performance and cost. The present model revealed that mean time to system failure decreases as pick and place failure rate increases and further, mean time to system failure has higher values for higher values of the rate at which PCB goes for inspection $(\gamma)$. Availability of the system decreases with increase in the values of different failure rates and increases with increase in value of respective repair rates. It can also be concluded that profit of the system increases with increase in the values of revenue per unit uptime whereas it decreases with increase in the values of cost per unit time of external repair, failure rate and shut down rate. Also, some cut off points for cost with respect to various parameters are obtained which helps the manufacturer to maintain optimal balance between cost, profit and quality.

\section{References}

[1] T. Nakagawa, S. Osaki,Stochastic behavior of a two-unit priority standby redundant system with repair,Microelectronics Reliability, 14,No.3 (1975),309-313 .

[2] M. Yamashem,A repairable multiple state device with general repair time,IEEE Transaction on Reliability, R-29, No.3 (1980), 276.

[3] L.R. Goel, A. Kumar, A. K. Rastogi,Analysis of a two-unit standby system with parallel and two types of repair,Microelectronics Reliability,24, No. 5(1984), 873-876.

[4] G. D. Goel, K. Murari, Two-unit cold standby redundant system subject to random checking, corrective maintenance and system replacement with repairable and nonrepairable types of failure, Microelectron Reliability, 30, No.4 (1990), 661-665. 
[5] M. N. Gopalan, K.S. Bhanu, Cost analysis of a two-unit repairable system subject to online preventive maintenance and/ or repair, Microelectronics Reliability, 35, No.2 (1995), 251-258.

[6] R. Gupta, Ram Kishan, R. Goel, Analysis of a system having super-priority, priority and ordinary units with arbitrary Distributions, Microelectron Reliability,37, No. 5 (1997), 851-856.

[7] K. H. Wang, C.C Kuo, Cost and probabilistic analysis of series system with mixed standby components, Applied Mathematical Modelling,24 , No. 12 (2000), 957-967.

[8] R. Kumar, U. Vashistha, R.K. Tuteja ,A two-unit redundant system with degradation replacement, Pure and Applied Mathematika Sciences 54, No. 1-2 (2001), 27-38.

[9] G. Taneja, J. Nanda, Probabilistic analysis of a two-unit cold standby system with resume and repeat repair policies, Pure and Applied Mathematika Sciences, 57, No.1-2 (2003), 3749.

[10] G. Taneja, V. Khurana, S.M. Rizwan, Economic analysis of a reliability model for twoprogrammable logic controller cold standby system with four types of failure, Pure Applied Mathematika Sciences, 63, No.1-2 (2006), 65-78.

[11] L. Xing, Reliability evaluation of phased-mission systems with imperfect fault coverage and common cause failures, IEEE Transactions on Reliability,56, No.1 (2007), 58-68.

[12] A. Goyal, G. Taneja, D.V. Singh,Analysis of a two-unit cold standby system working in a sugar mill with operating and rest period, Caledonian Journal of Engineering, Oman,5, No.1 (2009), 1-5.

[13] M.A.W. Mahmoud, M.E. Moshref,On a two-unit cold standby system considering hardware, human error failures and preventive maintenance, Mathematical and Computer Modelling, 51, No. 5 (2010), 736-745.

[14] R. Kumar, P. Bhatia,Reliability and cost analysis of one-unit centrifuge system with single repairman and inspection, Pure and Applied Mathematika Sciences, 74, No.(1-2) (2011), 113-121.

[15] A. Kumar, S.C.Malik,Reliability modelling of a computer system with priority to s/w replacement over h/w replacement subject to MOT and MRT, International Journal of Pure and Applied Mathematics, 80, No. 5 (2012), 693-709.

[16] R. Kumar, M. Kumar, Performance and cost benefit analysis of a hardware-software system considering hardware based software interaction failures and different types of recovery, International Journal of Computer Applications, 53, No.17 (2012), 25-32.

[17] R. Kumar, S. Batra,Economic and reliability analysis of a stochastic model on printed circuit boards manufacturing system considering two types of repair facilities, International Journal of Electrical Electronics and Telecommunication Engineering,43, No.10 (2012), 432-435.

[18] A. Hajeeh Mohammed, Availability of a system with different repair options, International Journal of Mathematics in Operational Research, 4, No.1 (2012), 41-55.

[19] M. Jain, S. Rani,Availability Analysis for Repairable System with warm standby, switching failure and reboot delay, Int. J. of Mathematics in Operational Research, 5, No.1 (2013), 19-39. 
[20] C.E. Wells, Reliability analysis of a single warm-standby system subject to repairable and non-repairable failures, European Journal of Operational Research, 235, No. 16 (2014), 180-186. 